\title{
Non-equispaced Fourier Transform vs. Polynomial-based Metal Artifact Reduction in Computed Tomography
}

\author{
Bärbel Kratz, Tobias Knopp, Jan Müller, May Oehler, Thorsten M. Buzug \\ Institute of Medical Engineering, University of Lübeck, 23538 Lübeck, Germany \\ kratz@imt.uni-luebeck.de
}

\begin{abstract}
We propose a method, which treats metal artifact reduction in CT by sinogram restoration as a problem of scattered data interpolation. Corrupted sinogram entries are discarded and replaced with artificially generated values. The presented interpolation algorithm is based on the 2D non-equispaced fast Fourier transform (NFFT). Results are compared to two common polynomial interpolation schemes.
\end{abstract}

\section{Introduction}

The non-equispaced fast Fourier transform (NFFT) [1] is a generalization of the fast Fourier transformation (FFT) for data located on non-equispaced grids. It has several applications in medical imaging, such as for instance it is used in magnetic resonance imaging for reconstruction of data located on spiral or radial trajectories. Further, NFFT can be used for direct reconstruction methods [2], exploiting the well known Fourier-slice theorem. Beside these applications, the NFFT can be generally used for multidimensional interpolation of scattered data as described in [3]. In the present work we propose to apply NFFT-based scattered data interpolation to metal artifact reduction in CT, where inconsistent data needs to be replaced by interpolated one.

If one or more metal objects, e.g. dental fillings or hip prosthesis are present in the field of view of a CT scanner, the reconstructed images are often severely degraded by bright star-shaped artifacts (Fig. 1). The main cause of these metal artifacts is the so called beam hardening effect, where photons of high and low energies are attenuated differently. Due to the highly non-linear nature of this process, this results in inconsistent projection data. These artifacts impare the diagnostic value of the reconstructed images and, therefore, give rise to the introduction of correction methods into the reconstruction schemes.

A common method for metal artifact reduction consists of two parts: First, the inconsistent data is removed from the sinogram. Afterwards, the resulting gap is to be filled with surrogate values. The first step can be accomplished by segmentation of the regions containing metal in a preliminary reconstruction. After forward projection of the resulting binary label image, the resulting mask can then be used to remove metal traces from the initial sinogram. In literature, 
several approaches to complete the sinogram are known. One popular method is proposed in [4] and [5], in which the gap is filled with interpolated values from adjacent detector elements. The interpolation is done independendly for each view.

In the present paper it is shown, how the computation of the discarded values can be interpreted as a problem of scattered data interpolation. Additionally it is shown, how this very problem can be solved using an NFFT based approach.

\section{Materials and Methods}

The FFT provides a method for interpolation of multidimensional data. By zero padding the vector of Fourier coefficients, the number of elements of an pixel grid can be increased. However, the FFT is limited to equispaced voxel positions and cannot be applied directly for interpolation of the gap in a sinogram. Therefore, we propose to use a generalization of the FFT, the so called NFFT that can handle non-equispaced voxel positions in the image domain. In the following we describe the application of NFFT interpolation to the gap filling problem in CT.

Let $p(\boldsymbol{r}), \boldsymbol{r}=(\theta, r)^{T}$ denote the measured projection that depends on the angle $\theta$ and on the position $r$. The projections are sampled on $N$ equispaced positions $\boldsymbol{r}_{\rho}$ that are located on a grid of size $N_{1} \times N_{2}$. For simplicity let the positions $\boldsymbol{r}_{\rho}$ be scaled to fit into the torus $\left[-\frac{1}{2}, \frac{1}{2}\right)^{2}$. After detection of metal traces, located on $N_{\text {gap }}$ positions $\overline{\boldsymbol{r}}_{n}$, only a subset of $M=N-N_{\text {gap }}$ projections are available located on non-equispaced positions $\tilde{\boldsymbol{r}}_{j}$.

In order to interpolate the missing values necessary for the reconstruction, a Fourier decomposition of the projections $p$ is performed, i.e. the complex coefficients $\hat{f}_{\kappa}$ are computed, such that

$$
p\left(\tilde{\boldsymbol{r}}_{j}\right)=\sum_{\kappa=0}^{N-1} \hat{f}_{\kappa} \mathrm{e}^{2 \pi \mathrm{i} \boldsymbol{k}_{\kappa}^{T} \tilde{\boldsymbol{r}}_{j}}, \quad \text { for } \quad j=0, \ldots, M-1
$$

where $\boldsymbol{k}_{\kappa} \in\left[-\frac{N_{1}}{2}, \frac{N_{1}}{2}\right) \times\left[-\frac{N_{2}}{2}, \frac{N_{2}}{2}\right)$ denote equispaced frequencies. Then, the missing values can be interpolated by evaluating (1) at the missing positions $\overline{\boldsymbol{r}}_{n}$
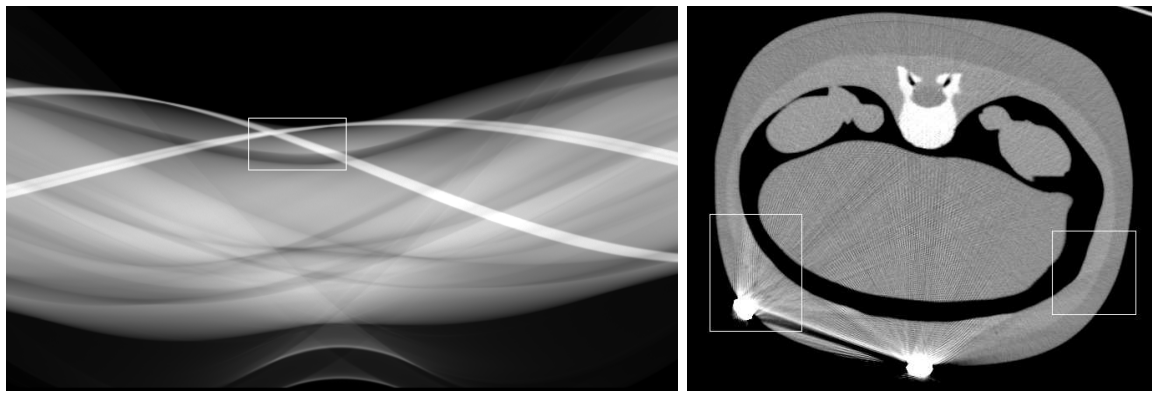

Fig. 1. Measured data (left) and reconstructed image (right) after filtered backprojection. Close-up regions for the results in Fig. 2 are marked 
for $n=0, \ldots, N_{\text {gap }}$. Turning (1) into matrix vector notation yields

$$
\boldsymbol{p}=\boldsymbol{A} \hat{f}
$$

with vectors $\boldsymbol{p}:=\left(p_{j}\right)_{j=0}^{M-1}, \hat{\boldsymbol{f}}:=\left(\hat{f}_{\kappa}\right)_{\kappa=0}^{N-1}$ and the non-equispaced Fourier matrix $\boldsymbol{A}:=\left(\mathrm{e}^{2 \pi \mathrm{i} \boldsymbol{k}_{\kappa}^{T} \tilde{\boldsymbol{r}}_{j}}\right)_{j=0, \ldots, M-1 ; \kappa=0, \ldots, N-1}$. Since $M<N$, the system (2) is underdetermined and, as proposed in [3], it can be solved by the minimization problem

$$
\|\hat{\boldsymbol{f}}\|_{\hat{\boldsymbol{w}}}^{2}=\sum_{\kappa=0}^{N-1} \frac{\left|\hat{f}_{\kappa}\right|^{2}}{\hat{w}_{\kappa}} \stackrel{\hat{\boldsymbol{f}}}{\rightarrow} \min \quad \text { subject to } \boldsymbol{p}=\boldsymbol{A} \hat{\boldsymbol{f}}
$$

where the damping factors $\hat{\boldsymbol{w}}=\left(\hat{w}_{\kappa}\right)_{\kappa=0}^{N-1}$ can be used to incorporate knowledge on the decay of the Fourier coefficients. In that way, we can choose a "smooth" solution of the under-determined system (2). As proposed in [3] the minimization problem (3) can be solved efficiently by a variant of the conjugated gradient (CG) method in combination with the NFFT. The CG algorithm proceeds iteratively performing multiple matrix-vector multiplications. Every matrix-vector multiplication can be performed by the NFFT, which is an approximative algorithm for the evaluation of (1) that needs only $\mathcal{O}(N \log N+\varepsilon M)$ operations, where $\varepsilon$ denotes the desired accuracy. In summary, the vector of Fourier coefficients $\hat{\boldsymbol{f}}$ can be calculated with $\mathcal{O}(\lambda(N \log N+\varepsilon M))$ operations, where $\lambda$ denotes the number of $\mathrm{CG}$ iterations. To compute the missing values from the Fourier coefficients $\hat{f}$, an ordinary FFT can be used.

To demonstrate the interpolation approach, the projection data of a slice through an anthromorphic torso phantom with two externally attached steel markers was used. The uncorrected dataset is shown in Fig. 1. In order to provide ground truth data for error assessment, the phantom was additionally scanned without markers. The image, which masks the metal traces, was generated by applying a simple threshold segmentation to a preliminary reconstructed image and a subsequent forward projection of this binary image.

Two different interpolation methods were applied for comparison with the proposed NFFT method: A linear and a cubic spline interpolation along single projections. For the implementation of the NFFT interpolation method, we use the software package described in [6]. The damping factors $\hat{\boldsymbol{w}}$ were chosen as the absolute value of the Fourier coefficients of the spline interpolated image. In this way, an appropriate weighting in Fourier space is enforced.

\section{Results}

The restorated sinograms and their corresponding reconstructed images are shown in Fig. 2. Visual inspection of the singoram data after interpolation suggests that the NFFT approach outperforms the two other methods. However, for regions in the reconstructed images in the vicinity of the metal markers, the spline based method seems to perform best. Streak artifacts situated further away from the markers are significantly reduced when using the NFFT approach. 
Fig. 2. Detailed view of the results. From top to bottom: Uncorrected dataset, a scan of the torso phantom without markers, linear sinogram interpolation, spline sinogram interpolation, and NFFT sinogram interpolation

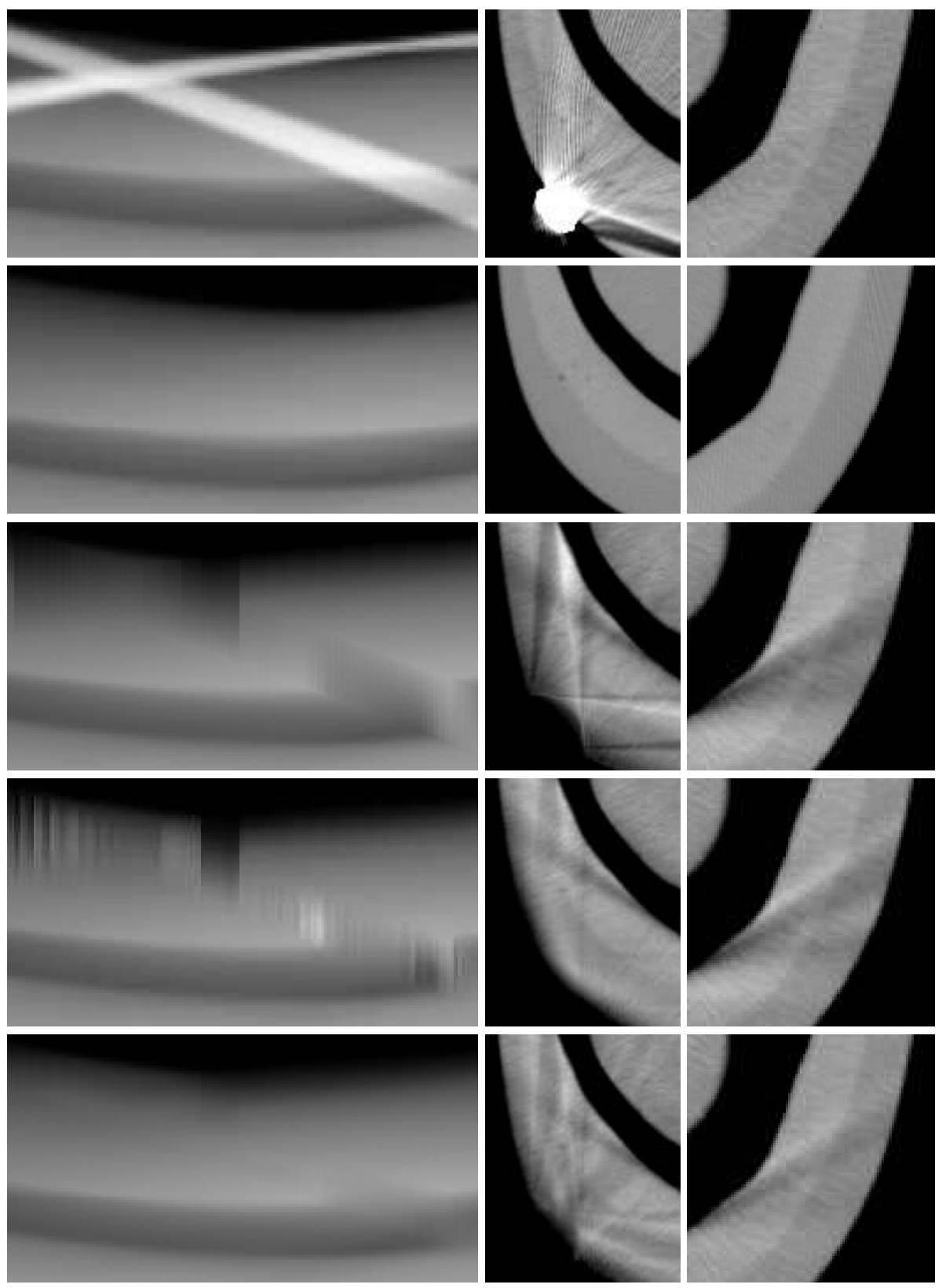


Table 1. Relative error of different interpolation methods

\begin{tabular}{cccc}
\hline Relative Error & Linear & Spline & NFFT \\
\hline Gap in Radon Space & 0.0681 & 0.0566 & 0.0316 \\
Image Space & 0.0978 & 0.0699 & 0.0668 \\
\hline
\end{tabular}

Linear interpolation performs worst in all cases. For error analysis the relative error $\|\boldsymbol{f}-\tilde{\boldsymbol{f}}\|_{2} /\|\boldsymbol{f}\|_{2}$ is computed, where $\boldsymbol{f}$ denotes the ground truth data and $\tilde{f}$ the data obtained by interpolation. For the sinogram data only the gap of missing values was taken into account for computation of the error. Results are listed in Tab. 1. As already seen in the close-up views, the NFFT provides the lowest error in Radon space, while the improvement for the reconstructed images is only marginal compared to the spline method.

\section{Discussion}

In the present work a new algorithm for metal artifact reduction based on twodimensional interpolation by means of non-equispaced Fourier transform was proposed. Missing sinogram values were filled with interpolated values, significantly reducing metal artifacts in the reconstructed images. For comparison, two polynomial algorithms were used. In the sinogram we achieved an improvement of the image quality using the NFFT interpolation. However, in the reconstructed images we obtained only a marginal improvement compared to spline based interpolation. This behavior may be due to the smoothness of the NFFT interpolation, since the filtered backprojection includes a high pass filtering. Taking this into account, an appropriate choice of the damping factors $\hat{\boldsymbol{w}}$ may yield better reconstruction results.

\section{References}

1. Potts D, Steidl G, Tasche M. Fast Fourier transforms for non-equispaced data: A tutorial. In: Modern Sampling Theory: Mathematics and Applications. Birkhäuser, Boston; 2001. p. 247-70.

2. Potts D, Steidl G. New Fourier reconstruction algorithms for computerized tomography. Proc SPIE. 2000;4119:13-23.

3. Kunis S, Potts D. Stability results for scattered data interpolation by trigonometric polynomials. SIAM J Sci Comput. 2007;29:1403-19.

4. Glover G H, Pelc N J. An algorithm for the reduction of metal clip artifacts in CT reconstructions. Med Phys. 1981;8(6):799-807.

5. Kalender W A, Hebel R, Ebersberger J. Reduction of CT artifacts caused by metallic implants. Radiology. 1987;164(2):576-7.

6. Keiner J, Kunis S, Potts D. NFFT3.0, Softwarepackage, C subroutine library; 2006. http://www.tu-chemnitz.de/ potts/nfft. 